\title{
MOTIVACIÓN, SATISFACCIÓN Y RENDIMIENTO: ¿EXISTEN DIFERENCIAS REALES ENTRE CIENCIAS Y LETRAS?
}

\section{MOTIVATION, SATISFACTION AND ACHIEVEMENT MOTIVATION: ARE THERE REALLY DIFFERENCES BETWEEN SCIENCES AND HUMANITIES?}

Inmaculada Concepción Muñoz Sánchez, Carmen Ramos Gómez, Armando Hinojosa Padilla y María Natalia Jiménez Cantero. Universidad de Granada

Artículo dirigido por: Inmaculada Valor Segura. Departamento de Psicología Social. Universidad de Granada

Fecha de recepción: 19 de marzo de 2014.

Fecha de revisión: 19 de marzo de 2014.

Fecha de aceptación: 11 de abril de 2014.

\section{Resumen}

Tras tanta polémica entre "letras" y "ciencias" sobre cuál de los dos es mejor o más difícil, se ha pretendido comprobar si existe algún tipo de diferencia entre ambas especialidades en cuanto a motivación, satisfacción o rendimiento; y a su vez, si existe algún tipo de relación entre estos tres constructos. Por lo tanto, participaron en el estudio una muestra de 120 alumnos de ambas ramas, obteniendo seis carreras diferentes con 20 alumnos en cada una. Los resultados no dieron ningún valor significativo en cuanto a diferencias entre ambas especialidades, pero sí una correlación entre motivación y satisfacción.

Palabras clave: Motivación de logro, satisfacción, rendimiento, carreras universitarias, estudio correlacional.

\begin{abstract}
After so much controversy between "Humanities" and "Sciences" about which one is better or harder, we'd like to test if there are any differences between both specialties in terms of motivation, satisfaction and academic achievement; and at the same time, if there is some kind of relationship between these three constructs. Thus, participated a sample of 120 students of those two specialities, from six different degrees, picking 20 students from each one. Results didn't provide any significant difference between both specialties, but did provide a correlation between motivation and satisfaction.
\end{abstract}

Key Words: Achievement motivation, satisfaction, academic achievement, university degree, correlational study. 


\section{Introducción}

Desde siempre ha habido una concepción general de que existen diferencias entre las distintas especialidades universitarias como letras y ciencias (Aunión, 2008). Esta concepción diferencial va desde la dificultad entre las distintas especialidades hasta el grado de motivación necesaria para realizarlas, pasando por el rendimiento obtenido por los estudiantes de dichas especialidades. Pero, ¿realmente existen tales diferencias, o se trata solamente de la competitividad? Para comprobar la veracidad o falsedad de este mito se ha realizado poca investigación en el ámbito nacional, dejando gran libertad para investigaciones pioneras como la que se describe a continuación.

Este estudio se ha realizado con el objetivo de comprobar si hay diferencias significativas entre las relaciones de las variables estudiadas (satisfacción, motivación de logro y rendimiento) según la pertenencia a una especialidad académica u otra.

Siguiendo la idea de Zubiri Sáenz (2013) con respecto a la satisfacción laboral, la satisfacción incide en la actitud de los estudiantes frente a sus obligaciones. Dicha satisfacción surge a partir de la correspondencia entre el trabajo real y las expectativas del alumno. Para aumentar el rendimiento se deberá tener presente tanto la motivación como los aspectos satisfactorios de los estudiantes.

Por otra parte, en el proceso de aprendizaje, la motivación se refiere a la implicación emocional e intelectual de los estudiantes por el hecho de considerarlo una experiencia interesante para su trayectoria vital (Masjuan, 2005). Dentro de este concepto existen dos variantes de motivación según si ésta se fundamenta en variables externas o internas a la persona (motivación extrínseca e intrínseca respectivamente). En este caso la motivación medida con los instrumentos utilizados se correspondería a uno de los casos de motivación extrínseca, Morales (2006) la define en términos de búsqueda del éxito, competitividad, aceptación de riesgos, constancia, organización en el trabajo y el proponerse objetivos a largo plazo (Aguilar de Tena, Mudarra Fernández, Perelló Alberola y Rojas González, 2013).

Por último, en este estudio el rendimiento se conceptualizó como una medida de las capacidades del alumno, que expresa lo que éste ha aprendido a lo largo del proceso formativo (Borgonovo, 2013). Gracias a diversos estudios se sabe que esta variable está relacionada con las dos anteriores de forma directa, de modo que una motivación insuficiente y una baja satisfacción dan lugar a un rendimiento pobre (Soler Porro y Chirolde Núñez, 2010).

Se pensó, por tanto que pueden existir diferencias entre motivación de logro, rendimiento y satisfacción dependiendo de la especialidad escogida de la misma forma que las hay de forma general, pues las dos especialidades escogidas tienen diferentes contenidos, modos de enseñanza, exigencias de aptitudes intelectuales, etc.

De esta forma se parte con la hipótesis de que habrá diferencias significativas entre las especialidades de ciencias y letras en la motivación de logro, el rendimiento y la satisfacción. 


\section{Método}

Participantes

La muestra se compuso de 120 estudiantes (39 hombres y 81 mujeres) de tercer año de diferentes carreras de la Universidad de Granada, con una media de edad de 22 años $(M=3,43)$. Se pidió la participación voluntaria de 20 alumnos de cada carrera: Estudios ingleses, Derecho, Filosofía, Enfermería, Biología y Farmacia. Dividimos estas carreras en especialidades generales, es decir, en Letras (Estudios ingleses, Derecho, Filosofía) y Ciencias (Enfermería, Biología y Farmacia).

\section{Diseño}

Hemos seguido un diseño correlacional, en el que se han medido varios constructos hipotéticos: motivación de logro, satisfacción y rendimiento; así como variables sociodemográficas: edad, sexo, carrera, curso y turno.

\section{Materiales}

Para la recogida de datos se elaboró un cuestionario con la siguiente estructura:

- Datos sociodemográficos: se les pedía que indicaran su edad, sexo, carrera, curso y turno.

- Rendimiento: se preguntó por la nota media del expediente sobre 10.

- Satisfacción: se utilizó el cuestionario SEUE de Palacios y García (2003) para conocer la satisfacción de los estudiantes universitarios con su educación.

- Motivación de Logro: se utilizó una adaptación de la "Escala de motivación de logro (ML-1)" (Brown, Cherrington y Cohen, 1975; Mehrabian, 1968).

\section{Procedimiento}

Se solicitó el permiso al profesor correspondiente para que diera acceso a la clase durante 15 minutos. Una vez conseguidos 20 alumnos voluntarios, repartimos los cuestionarios indicando que era anónimo y no había respuestas correctas ni incorrectas. Los investigadores estuvieron a la disposición de los alumnos por si tenían alguna duda.

\section{Resultados}

Se realizaron diferentes análisis estadísticos: descriptivos, correlaciones (Pearson) y prueba $\mathrm{T}$ para muestras independientes. Estos análisis se efectuaron con el programa estadístico SPSS 19.0. 
En cuanto al análisis descriptivo de los tres constructos hipotéticos, se puede apreciar como no hay grandes diferencias de puntuación entre letras y ciencias en cada uno de ellos.

Tabla 1. Análisis descriptivos. Estadísticos de grupos

\begin{tabular}{lllcc}
\hline & Especialidad & $\mathrm{N}$ & $\mathrm{M}$ & $\mathrm{DT}$ \\
\hline \multirow{2}{*}{ Rendimiento } & Ciencias & 59 & 7,01 & 1,43 \\
\cline { 2 - 5 } & Letras & 59 & 6,92 & 1,38 \\
\hline \multirow{2}{*}{ Satisfacción } & Ciencias & 60 & 274,70 & 47,40 \\
\cline { 2 - 5 } & Letras & 60 & 275,60 & 53,98 \\
\hline \multirow{2}{*}{ Motivación } & Ciencias & 60 & 40,63 & 5,59 \\
\cline { 2 - 5 } & Letras & 60 & 41,63 & 6,92 \\
\hline
\end{tabular}

Con el objetivo de estudiar si hay correlación entre los distintos constructos utilizados, se realizó una correlación entre ellos, que nos muestra una correlación directa entre motivación de logro y satisfacción ( $r=.258)$. Si realizamos esta misma correlación diferenciando entre las especialidades de las carreras (letras y ciencias) solo encontramos esta correlación entre satisfacción y motivación con los alumnos de letras.

Tabla 2. Correlaciones bivariadas entre las diferentes variables

\begin{tabular}{|c|c|c|c|c|}
\hline & \multicolumn{2}{|c|}{ RENDIMIENTO } & \multicolumn{2}{|c|}{ SATISFACCIÓN } \\
\hline & Letras & Ciencias & Letras & Ciencias \\
\hline SATISFACCIÓN & -.104 & $.283^{*}$ & -- & --- \\
\hline $\begin{array}{c}\text { MOTIVACIÓN DE } \\
\text { LOGRO }\end{array}$ & .024 & .152 & .223 & .088 \\
\hline
\end{tabular}

Nota. ${ }^{*} p<0,05$

En las pruebas $t$ para muestras independientes realizadas, los resultados no mostraron diferencias significativas en rendimiento, satisfacción y motivación de logro en función de la carrera.

\section{Discusión}

Tras el análisis de los resultados, se ha encontrado una correlación positiva entre satisfacción y motivación de logro, por lo que, en general, aquellos alumnos que tenga mayor satisfacción, también tendrán una mayor motivación. En cuanto al rendimiento se ha encontrado una relación por lo que hay una tendencia a que exista mayor rendimiento a mayor motivación (Masjuan, 2005).

En cuanto a las diferentes especialidades, se ha encontrado una relación entre satisfacción y rendimiento de los estudiantes de ciencias, pero no en la otra 
especialidad ni en los otros constructos. Una posible causa de esto, podría ser que la muestra no sea lo suficientemente representativa de la población al estar compuesta por pocas personas. También ha podido afectar que los estudiantes escogidos son, por lo general, de cursos avanzados, por lo que deben tener una motivación mayor para continuar con sus estudios. Otro factor a tener en cuenta es si las carreras escogidas son lo suficientemente representativas de las especialidades que se escogieron. Otra variables, como la categoría socio-profesional (MasJuan, 2005), podrían influir en el estudio debido a su relación con la motivación, por lo que deberían haberse tenido en cuenta. Sería interesante replicar el estudio con distintos cursos de una misma carrera y carreras más significativas dentro de cada especialidad.

Un posible problema de nuestro estudio podría ser el límite de tiempo impuesto por algunos profesores a la hora de realizar el cuestionario. También debería tenerse en cuenta el hecho de que la mayoría de nuestra muestra está compuesta por mujeres, lo que podría afectar si hubiera diferencias entre ambos sexos, ya que los participantes participaron de manera voluntaria.

Hoy en día, hay un gran número de abandonos de carreras, en algunas titulaciones hasta un $50 \%$, debido a que los estudiantes no han escogido correctamente aquella que coincide con su vocación. Hasta un tercio de los alumnos de primer año, consideran seriamente abandonar su titulación (González Alfonso, Álvarez Pérez, Cabrerar Pérez y Bethencourth Benítez, 2007). El psicólogo está disponible, desde secundaria, para ayudar a los alumnos en su futuro académico, orientándolos hacía el ámbito académico y laboral que les produzcan una mayor motivación de logro, ya que esto aumentará su satisfacción y, probablemente, su rendimiento (Ramírez et al., 2013; Soler Porro et al., 2010); lo cual también reducirá el abandono.

Si en futuras investigaciones, nuestras hipótesis se corroboran, se podrá afirmar -de lo cual se está tomando ya consciencia- que el psicólogo tiene un papel fundamental en el ámbito académico. Tal vez sería interesante la posible incorporación de la figura del psicólogo al ámbito universitario, otorgándole un papel de ayuda y orientación, de cara a mejorar las expectativas de futuro profesional y aumentar la percepción de motivación de logro (Aguilar de Tena et al., 2013). Nuestro estudio es solo un acercamiento a la problemática, que aportar más evidencias en este tipo de investigación.

\section{Referencias}

Aguilar de Tena, P., Mudarra Fernández, R., Perelló Alberola, S. y Rojas González, C. (2013) Ansiedad y motivación de logro en estudiantes universitarios: un estudio correlacional. ReiDoCrea, 2, 11-16.

Aunión, J.A. (2008). Vago, a letras; empollón, a ciencias. Recuperado de http://elpais.com/diario/2008/06/30/sociedad/1214776807_850215.html del 30 de junio

Borgonovo, D. B. (2013). Deseo de saber y rendimiento académico. Puntos de encuentro. Tesis de maestría no publicada, Universidad FASTA, Mar del Plata, Argentina.

Brown, G., Cherrington, D. H., y Cohen, L. (1975). Experiments in the Social Science. London: Harper and Row. 
Cabrera Guillén, P. y Galán Delgado, E. (2002). Factores contextuales y rendimiento académico. Revista Electrónica Interuniversitaria de Formación del Profesorado, 5(3).

Cryer, P. y Elton, L. (1990). Catastrophe Theory: a unified model for educational Change. Studies in Higher Education, 15(1), 75-87.

Fortier, M.S., Vallerand, R.J., y Guay, F (1995). Academic Motivation and School Performance: Toward a Structural Model. Contemporary Educational Psychology, 20, 257-274.

González Alfonso, M.C., Álvarez Pérez, E.R., Cabrerar Pérez, L. y Bethencourth Benítez, J. T. (2007). El abandono de los estudios universitarios: factores determinantes y medidas preventivas. Revista española de pedagogía, 236, 7186.

Masjuan, J.M. (2005). Progresos en los aprendizajes, características de los estudios y motivaciones de los estudiantes. Papers, 76, 97-133.

Morales, P. (2006). Medición de actitudes en Psicología y Educación; construcción de escalas y problemas metodológicos. Madrid: Universidad Pontificia Comillas.

Palacios, S. y García, M. (2003). EI SEUE: un instrumento para conocer la satisfacción de los estudiantes universitarios con su educación. Acción pedagógica, 12(2), 16-27.

Ramírez, P.E. y Fuentes, A.C. (2013) Felicidad y rendimiento académico: efecto moderador de la felicidad sobre indicadores de selección y rendimiento académico de alumnos de ingeniería comercial. Formación Universitaria, 6 (3), 21-30.

Soler Porro, A.B. y Chirolde Núñez, R.R. (2010) Motivación y rendimiento docentes en estudiantes bolivianos del Nuevo Programa de Formación de Médicos. Educación Médica Superior, 24 (1), 42-51.

Zubiri Sáenz, F. (2013) Satisfacción y motivación profesional. Anales del sistema sanitario de Navarra, 36 (2), 193-196. 